\title{
Identifying Attenuating Mutations: Tools for a New Vaccine Design against Flaviviruses
}

\author{
Cécile Khou Nathalie Pardigon \\ Arbovirus Group, Environment and Infectious Risks Unit, Institut Pasteur, Paris, France
}

\section{Keywords}

Flavivirus vaccines - Molecular infectious clones - Live attenuated virus strains - MicroRNA target sequences

\begin{abstract}
Emerging Flaviviruses pose an increasing threat to global human health. To date, human vaccines against yellow fever virus (YFV), Japanese encephalitis virus (JEV), dengue virus (DV), and tick-borne encephalitis virus (TBEV) exist. However, there is no human vaccine against other Flaviviruses such as Zika virus (ZIKV) and West Nile virus (WNV). In order to restrict their spread and to protect populations against the diseases they induce, vaccines against these emerging viruses must be designed. Obtaining new live attenuated Flavivirus vaccines using molecular biology methods is now possible. Molecular infectious clones of the parental viruses are relatively easy to generate. Key mutations present in live attenuated vaccines or mutations known to have a key role in the Flavivirus life cycle and/or interactions with their hosts can be identified by sequencing, and are then inserted in infectious clones by site-directed mutagenesis. More recently, the use of chimeric viruses and large-scale reencoding and introduction of microRNA target sequences have also been tested. Indeed, a combination of these methods will help in designing new generations of vaccines against emerging and reemerging Flaviviruses.

(c) 2017 S. Karger AG, Basel
\end{abstract}

\section{Introduction}

Flaviviruses are arthropod-borne (arbo)viruses transmitted through the bite of mosquitoes or ticks [1]. They pose an increasing threat to global human health [2-5]. Live attenuated vaccines against yellow fever virus (YFV) and Japanese encephalitis virus (JEV) exist $[6,7]$. A live attenuated vaccine against tick-borne encephalitis virus (TBEV) was produced in 1937; however, due to the high risk of adverse events following vaccination, only inactivated TBEV vaccines are used today [8]. The use of infectious clones generated by molecular biology techniques opened new possibilities to design vaccinal viral strains. Indeed, a tetravalent vaccine against dengue virus (DV) has recently been designed $[9,10]$.

However, no human vaccine is yet available against other Flaviviruses such as West Nile virus (WNV) or Zika virus (ZIKV). Mostly a zoonotic pathogen, WNV may kill birds and induce encephalitis in horses and humans [1113]. While a vaccine exists to protect horses [14], there is no human vaccine against WNV although the virus has killed $>2,000$ people since its introduction into the USA in 1999 [15], and it is still responsible for outbreaks in the human population in Europe and Africa [16]. ZIKV was responsible for a massive epidemic in the Western hemisphere in 2014-2016 [17], with $>2,000$ recorded cases of congenital malformations and at least 100 cases of auto-

Nathalie Pardigon

Arbovirus Group, Environment and Infectious Risks Unit, Institut Pasteur 25 , Rue du Docteur Roux

FR-75015 Paris (France)

E-Mail pardigon@pasteur.fr 
immune neuropathy (Guillain-Barré syndrome) $[18,19]$. A vaccine against ZIKV would certainly be welcome as well. As more pathogenic arboviruses will most likely emerge/reemerge in the near future, and amongst them Flaviviruses, we must be prepared to respond to new vaccinal needs.

Flaviviruses are enveloped viruses with a singlestrand, positive-sense RNA genome encoding a polyprotein that is co- and posttranslationally cleaved by cellular and viral proteases into 3 structural (capsid [C], premembrane $[\mathrm{prM}]$, and envelope $[\mathrm{E}]$ ) and 7 nonstructural (NS1, NS2A, NS2B, NS3, NS4A, NS4B, and NS5) proteins. The structural proteins assemble to produce virus particles, while the nonstructural proteins play a role in viral replication and immune evasion [20]. Interestingly, and as with other viruses with RNA genomes, a given Flavivirus is not a clonal population of a virus, but rather a mix of multiple viral genomic species (quasispecies) due to the Flavivirus error-prone NS5 polymerase that can frequently introduce mutations in the viral genome during replication. Thus, a Flavivirus genomic sequence represents a consensus sequence between that of all its quasispecies [21,22]. These populations of closely related viral variants result in genetic diversity that may affect both pathogenesis and therapeutic responsiveness [23].

Although the Flavivirus genomic arrangement and sequence were not known at the time, live attenuated strains of YFV were obtained empirically in the 1930s and used as vaccines [6]. JEV vaccine was also obtained empirically [24]. How these vaccine strains are attenuated remains unclear. Molecular tools are now used to decipher the bases of viral attenuation and to generate new vaccinal Flavivirus strains.

\section{Live Attenuated Vaccines}

\section{YFV Vaccines}

The most efficient vaccines against Flaviviruses are YFV vaccines. They were obtained empirically in the 1930s [6].

The YFV FNV (French neurotropic virus) vaccine was obtained in 1930 via several passages of the virulent YFV FVV (French viscerotropic virus) strain on mouse brain. However, due to the high rates of postvaccinal encephalitis, the use of this vaccine was discontinued in 1982 [6]. YFV FNV vaccine has 35 mutations in the amino acid sequence [25] when compared to that of the virulent FVV strain.

Tools for Flavivirus Attenuation
YFV 17D vaccinal strain was obtained in 1937 by several passages of the virulent YFV Asibi strain on sucklingmouse brain and chick embryo tissues [6]. It has 31 mutations in the amino acid sequence and 4 mutations in the $3^{\prime}$ UTR [26] compared to that of the virulent Asibi strain. Curiously, YFV 17D and YFV FNV vaccines have only 2 nonsynonymous mutations in common: M-I36F and NS4B-I95M $[25,26]$.

Currently, the correlation between attenuation and the amino acid sequence is not understood. However, it seems that apart from the observed mutations, loss of quasispecies diversity in the YFV $17 \mathrm{D}$ vaccinal strain may also participate in its attenuation [26].

\section{The JEV SA14-14-2 Vaccine}

Another vaccine that was obtained empirically is the JEV vaccine SA14-14-2. It was obtained by several passages of virulent JEV SA14 strain on hamster kidney PHK cells and chick embryo PCE cells [24]. It has $25 \mathrm{mu}-$ tations in the amino acid sequence, 1 in the $5^{\prime}$ UTR and 1 in the $3^{\prime}$ UTR when compared to that of JEV SA14 strain [27]. Among these mutations, those in the $5^{\prime}$ UTR, in the capsid protein, and in the NS1-NS2A region reduce neuroinvasiveness. Furthermore, mutations in the E protein induce complete attenuation of neurovirulence of JEV SA14-14-2 strain [28]. Particularly, it has been shown that reverse mutation G244E in the E protein of JEV SA14-14-2 vaccine induces lethal neurovirulence in mice. The E-244 amino acid is in a hairpin near the $\mathrm{E}$ protein fusion loop. It has been shown that mutation E244G in JEV SA14-14-2 impairs early steps - binding, endocytosis, and membrane fusion - of the virus life cycle in neuronal cells, and possibly the late steps - assembly, maturation, and release - of the virus life cycle [29].

Synonymous mutations may also play a role in the attenuation of JEV SA14-14-2 vaccinal strain. Members of JEV serocomplex have a conserved pseudoknot structure in their genomic RNA. This structure induces a ribosomal frameshifting during the translation of the viral genome, leading to the secretion of a protein called NS1'. This protein is linked to viral pathogenesis, especially viral neuroinvasiveness [30]. JEV SA14-14-2 vaccinal virus has a G66A mutation in the NS2A protein coding sequence. This mutation may destabilize the conserved pseudoknot structure, resulting in the suppression of the ribosomal frameshifting that in turn leads to the suppression of the NS1' protein expression. This suppression seems to contribute to viral attenuation [31].

Intervirology 2017;60:8-18

DOI: $10.1159 / 000479966$ 


\section{Vaccines}

Attenuated DV strains against the 4 different DV serotypes have also been obtained empirically. The DV1 attenuated strain MD-1 has been obtained by several passages on mouse brain [32]. Two DV2 attenuated strains, DV2 PR-159 S-1 and DV2 16881 PDK-53, were obtained by plaque purification on primary green monkey kidney cells of DV2 PR-159 strain [33] and via several passages of DV2 16881 strain on primary dog kidney cells [34]. DV3 16562 PGMK 33 and DV4 H-241 PDK 35 attenuated strains were obtained via several passages of DV3 16562 strain on primary green monkey kidney cells [35] and DV4 H-241 strain on primary dog kidney cells [36].

A major obstacle for DV immunization is the antibody-dependent enhancement (ADE) phenomenon. This phenomenon was first observed in 1980 in Thailand. Epidemiology studies showed that secondary infections with a given DV serotype following infection with another DV serotype enhance the risk of developing a severe form of dengue hemorrhagic fever [37]. These observations were confirmed on mouse macrophage-like cells, where infection by DV2 was potentiated with monoclonal antibodies against DV4 [38]. Due to this ADE phenomenon, simultaneous vaccination against each DV serotype must be performed to induce full protection against all 4 serotypes of DV. A tetravalent vaccine prototype has been tested in humans with 4 live attenuated viruses obtained via several passages in primary dog kidney cells and primary green monkey cells. Vaccinated subjects developed symptoms following vaccination, and only DV3 attenuated virus was detected in their blood, suggesting that this virus is more replicative than the other 3. Regarding the induction of protective immunity, all subjects vaccinated with the tetravalent vaccine produced antibodies against DV3. However, only some of them produced antibodies against the other 3 serotypes [39].

\section{Killed Vaccines}

\section{TBEV Vaccines}

Following the protocol by which the YFV-17D vaccine was obtained, a live attenuated vaccine against TBEV was produced by passage in suckling-mouse brain in 1937 . However, due to the high rates of adverse events following vaccination, this vaccine is no longer used [8]. Inactivated vaccines are used instead. To date, there are 4 different inactivated vaccines against TBEV.

\section{Molecularly Engineered Vaccines}

\section{Chimeric Vaccines That Use Live Attenuated}

Flavivirus Vaccine Genomes as a Backbone

The development of infectious clones has allowed the development of molecularly engineered vaccines. Infectious clones are DNA plasmids or linear DNAs that encode a viral genome and that, once transfected in cells, will be transcribed and translated, leading to the production of infectious viral particles. Molecularly engineered viral genomes can thus be produced. These genomes can contain coding regions originating from different viruses, leading to "chimeric" viruses. The highly immunogenic viral proteins $\mathrm{E}$ and prM are particularly interesting for introduction into these "chimeric" viruses as they induce a strong host immune response [40, 41].

As Flaviviruses have the same genome organization and replication features, designing "chimeric" viruses using an attenuated Flavivirus genome as a vector can be a tool to design new vaccine candidates. For example, the ChimeriVax method used to design new Flavivirus vaccines consists of using a molecular infectious clone of the YFV 17D vaccine genome as a backbone, and then replacing the genome region encoding YFV 17D prM and $\mathrm{E}$ proteins by that of the target Flavivirus that the vaccine is designed against [42]. Such viral chimera should demonstrate an attenuated phenotype when compared to that of the target virus and thus induce an immune protection against it [42].

Currently, 2 vaccines based on this method are commercially available. A first vaccine against JEV, Imojev, encodes the prM and E proteins of the JEV SA14-14-2 vaccinal strain [42]. The DV CYD-TDV dengue vaccine, Dengvaxia, is the second licensed vaccine based on the ChimeriVax method. The genome region encoding the prM and E proteins of each DV serotype replaces that of YFV 17D, leading to 4 chimeras representing each serotype of DV [9].

Using the same ChimeriVax method, a new vaccine prototype has been proposed for WNV (ChimeriVaxWN02). It encodes WNV flamingo isolate 388-399 prM and $\mathrm{E}$ proteins. Additional mutations identified in JEV SA14-14-2 vaccine genome, L107F, A316V, and K440R in the $\mathrm{E}$ protein, were introduced in this chimeric vaccine to reduce neurovirulence of the ChimeriVax-WN vaccine prototype, thus increasing the safety of the prototype [43].

Other Flavivirus live attenuated vaccine genomes have also been used to produce chimeric vaccines. The JEV SA14-14-2 genome has been used to design vaccines
10

Intervirology 2017;60:8-18

DOI: $10.1159 / 000479966$
Khou/Pardigon 
against DV1, DV2, DV4, and TBEV by replacing the prM and $\mathrm{E}$ protein coding regions by those of the targeted virus [44-47].

Likewise, 2 different live attenuated strains of DV were used to design new vaccine candidates, DV2 16881 PDK53 vaccine and recombinant DV4 814669, where $30 \mathrm{nu}-$ cleotides were deleted from the 3'UTR $[34,48]$. So far, vaccine prototypes against DV1, DV3, DV4, and WNV using DV2 16881 PDK-53 vaccine genome as a backbone have been produced. Two methods have been tested: one consisting of replacing the $\mathrm{C}$, prM, and $\mathrm{E}$ protein coding regions by those of the targeted virus [49], and the other by replacing only the prM and $\mathrm{E}$ protein coding regions $[50,51]$. Using the recombinant DV4 814669 live attenuated virus genome [48] as a backbone, vaccine prototypes against TBEV, WNV, and JEV have been designed by replacing the prM and $\mathrm{E}$ protein coding regions by those of the targeted virus [52-54]. For the JEV vaccine prototype, additional mutation of the E protein E-Q264H was introduced, and the resulting virus was shown to replicate to higher levels in tissue culture and was more immunogenic [54].

\section{Chimeric Vaccines: Measles Virus Schwarz Strain and Lentivirus as Vectors}

Using vaccinal strains of viruses from other viral families as vectors to engineer chimeric viruses expressing structural proteins of a given Flavivirus is another interesting possibility, in particular in view of pediatric vaccination [55]. For example, the measles virus (MV) Schwarz vaccinal strain is a safe and efficient human vaccine, the genome of which can be used as a vector [55]. The WNV IS98-ST1 strain E protein coding region has been introduced in an MV infectious clone, allowing secretion of WNV E protein following infection by the resulting chimeric virus. Mice infected with this virus produced antibodies against WNV $[56,57]$. A new vaccine candidate against DV has also been developed using this method. The coding region of domain III of the E protein (i.e., the Flavivirus receptor-binding domain), belonging to 4 different DV serotype strains was fused to their respective $M$ protein ectodomain and introduced into a MV infectious clone [58]. Intriguingly, adding the M protein ectodomain to the $\mathrm{E}$ protein domain III was found to enhance host cytokine secretion and thus antiviral immune response [59]. Mice infected with the resulting virus produced antibodies against each DV serotype [60].

Tools for Flavivirus Attenuation
Another vector used in the design of new vaccines is the nonintegrative lentiviral vector TRIP [61]. The WNV E protein coding region was introduced into this lentiviral vector. Infection by the resulting lentivirus induced the secretion of WNV E protein. Mice infected with this recombinant lentivirus produced antibodies against WNV [62]. The JEV prM and E protein coding regions were also used in another study; the resulting lentivirus induced a broad neutralizing antibody response in pigs [63].

\section{Designing New Molecularly Cloned Vaccines: Live Attenuated Vaccines as a Source of Inspiration}

The use of infectious clones also permits the development of mutant attenuated viruses using site-directed mutagenesis. Introducing known mutations of the 2 live attenuated vaccines against YFV and JEV into molecular clones of different Flaviviruses may help in the design of new molecularly cloned vaccines. For example, the residue in position 36 of the $\mathrm{M}$ protein (part of the M protein ectodomain, a relatively well conserved region among Flaviviruses) is a leucine in the YFV strain Asibi, but a phenylalanine in the 2 vaccinal strains 17D and FNV [25, 26]. An isoleucine to phenylalanine (I36F) substitution was introduced into the $\mathrm{M}$ ectodomain region of a JEV molecular clone $[64,65]$. The resulting mutant virus life cycle was impaired in mammalian cells but not in mosquito cells. Furthermore, it was attenuated in a mouse model of JEV pathogenesis, and could induce the production of neutralizing antibodies against JEV, suggesting that such a substitution could be included in the design of a new JEV molecularly cloned, live attenuated vaccine [65]. However, when the corresponding I36F substitution was introduced into the YFV Asibi strain, the proapoptotic effect of YFV Asibi M protein ectodomain was suppressed [66]. In mammalian cells, the viral replication was not impaired [67].

Other studies have experimented with introducing mutations of the JEV SA14-14-2 vaccine strain into the WNV genome, another member of the JEV serocomplex. The introduction of the mutations L107F, E138K, Y172V, $\mathrm{T} 173 \mathrm{~A}$, and $\mathrm{A} 312 \mathrm{~V}$ into the E protein coding region [68] of WNV molecular clones resulted in the attenuation of the WNV virus in a mouse model of pathogenesis. Mutations GCE AAG to GCE AA므 of codons 30/31 of WNV NS2A protein that suppress the conserved pseudoknot RNA structure in the NS2A protein coding region (essential for the translation and secretion of the NS1' protein)

Intervirology 2017;60:8-18 
resulted in a viral mutant unable to secrete the NS1' protein. Interestingly, this mutant was attenuated in the mouse model of pathogenesis [30].

\section{Impairing Virus Interactions with the Host Cell}

Instead of relying on mutations that were found in attenuated flaviviral strains, attenuation may be obtained by introducing mutations that may impair virus interactions with the host cell, in particular its innate immune response.

Alpha/beta interferons (IFN- $\alpha / \beta)$ are recognized cellular mediators of the innate immune response against flaviviral infections $[69,70]$. The recognition of viral RNA by RIG-I and PI3K followed by NF- $\kappa$ B protein expression eventually results in the activation of the interferon immune response [71]. To escape the cellular immune response, Flaviviruses have developed different strategies. One of these is to mimic cellular mRNA by capping viral RNA [72]. The viral RNA capping is performed by NS3 and NS5 proteins [73]. Targeting the $2^{\prime}$-O-methylation activity of these proteins may alter the flaviviral riposte to the cellular antiviral response. Mutation E218A in the NS5 methyltransferase active site of the JEV NS5 protein impairs the $2^{\prime}$-O-methylation of the JEV viral RNA cap. The resulting mutant virus is more sensitive to the antiviral effects of interferon and interferon-induced genes, such as interferon-inducible protein with tetratricopeptide 1 (IFIT1). IFIT1 was shown to bind preferentially to unmethylated mRNA, leading to the restriction of mRNA translation [74]. Thus, the translation of the unmethylated JEV NS5-E218A mutant genome was impaired; this mutant was attenuated in a mouse model of JEV pathogenicity and induced a protective immune response against JEV [75]. This method of virus attenuation can also be used for WNV and DV [76].

Flavivirus nonstructural proteins have also been found to directly inhibit IFN- $\beta$ transcription $[77,78]$. To identify mutations that can lead to more efficient production of IFN- $\beta$, persistent noncytopathic replication of flaviviral replicon RNAs (truncated flaviviral genomes that lack their structural genes, but retain their replicative ability) can be generated. Mutations responsible for adaptation of the replicons can then be identified and introduced into a molecular infectious clone [77]. For example, the A30P mutation in the NS2A protein of WNV was found to suppress the inhibitory effect on IFN- $\alpha / \beta$ production by the NS2A protein. The resulting mutant virus was attenuated in an in vivo model of viral pathogenesis [79]. Interest- ingly, this mutation also affects the pseudoknot RNA secondary structure, leading to the expression of NS1' protein. Western blot analysis showed that the mutant virus NS2A-A30P does not express NS1' protein. When compared to an attenuated mutant, which does not express the NS1' without affecting the protein sequence of the NS2A protein, it was found that the NS2A-A30P mutant virus was more attenuated, suggesting that attenuation of this mutant virus is due to 2 independent effects [30].

Flavivirus structural proteins also interact with the host cell. Impairing these specific interactions by introducing mutations in the structural proteins may lead to virus attenuation. By passaging the virus several times in cells incubated with an excess of receptor, mutations in the viral surface proteins can be identified. Wild-type viruses still binding this receptor will not be able to interact with the cell receptors, and so will not be able to infect the cells whereas mutant viruses that cannot bind this receptor will infect the cells by binding with another cell receptor. These mutant viruses will then be selected after several passages, and the mutations essential to receptor binding can be identified. For example, JEV mutant EG306E was obtained by passages in C6/36 cells incubated with an excess of membrane receptors isolated from a mouse brain. This mutant does not recognize the membrane receptors and was attenuated in a mouse model of JEV pathogenicity [80].

Instead of relying on viral adaptation to specific culture conditions to identify mutations that can impair interaction with receptors, bioinformatics analysis can be performed to identify potential locations for interesting mutations. Based on protein structure, the TBEV E$\mathrm{T} 310 \mathrm{~K}$ mutation was identified in the $\mathrm{E}$ protein domain III. This domain is involved in receptor recognition [58, 81]. The mutation E-T310K was an attenuating mutation in a mouse model of TBEV pathogenicity [82].

Bioinformatics analysis of protein amino acid sequences can also be used to identify canonic motif of domains interacting with specific receptors. For example, the integrin-binding RGD canonic motif was identified in Murray Valley encephalitis virus E protein. Mutating this motif by introducing mutations E-D390N, E-D390E, and E-D390Y leads to virus attenuation [83]. These mutations impede E protein binding to integrin but also increase its affinity to glycosaminoglycans (GAG) [84]. This increase in GAG affinity can also be achieved by several passages on human adrenal gland/cortex SW13 cells $[85,86]$. When injected into mice susceptible to viral infections, the mutant virus is rapidly cleared from the bloodstream. This may be due to a more rapid entry and infection of
12
Intervirology 2017;60:8-18 DOI: $10.1159 / 000479966$
Khou/Pardigon 
peripheral organs due to the increased $\mathrm{E}$ protein affinity to GAG present on the surface of blood vessel endothelial cells. Following the infection of peripheral organs, the virus can be eliminated. Overall, the increase of the E protein affinity to GAG leads to an attenuation of the mutant virus $[85,86]$. Interestingly, it was shown that the mutation E-T380R, present in YFV 17D vaccine, increases the affinity of its E protein to GAG, suggesting a role of this increased affinity in the attenuation of YFV 17D vaccine [87].

Structural proteins are not only involved in interactions with cellular receptors during the early steps of viral entry into the host cell, but also play a major role in the late stages of viral assembly and secretion. Introducing mutations that can impair these stages can lead to virus attenuation. For example, when mutation M-I36F was introduced in the region encoding the $\mathrm{M}$ protein in a JEV infectious clone, virus assembly and/or secretion was impaired in mammalian cells. The mutant virus M-I36F was also attenuated in a mouse model of JEV infection [65].

\section{Targeting the N-Glycosylation Sites of the prM, E, and NS1 Proteins}

The glycosylation status of Flavivirus glycoproteins prM, E, and NS1 is important for their interaction with host cells [88]. N-glycosylation of the prM and E proteins is essential for the proper assembly and secretion of virus particles [89-92]. The mutating prM putative N-glycosylation site was shown to impair the late stages of the virus life cycle and to affect the pathogenicity in murine models [91].

The E protein N-glycosylation status was a determinant of viral neuroinvasiveness in a mouse model of Flavivirus infection [93]. Indeed, the pathogenicity of neuropathogenic Flaviviruses, such as WNV and JEV, relies not only on their replicative capacity in the brain, but also on their ability to cross the blood-brain barrier in order to infect the targeted neuronal cells $[94,95]$. Deleting the E protein $\mathrm{N}$-glycosylation site impedes neuropathogenic WNV and JEV viruses from entering the central nervous system and infecting the target neuronal cells. Thus, mutant WNV and JEV viruses devoid of E protein N-glycosylation site do not induce neuropathogenic diseases [96-98].

$\mathrm{N}$-glycosylation of NS1 protein is also important in the pathogenicity of Flaviviruses in addition to the role this protein plays in Flavivirus replication. The hexameric

Tools for Flavivirus Attenuation form of DV NS1 protein interacts with the cellular complement, contributes to DV pathogenicity, and leads to the immune evasion of the virus [99]. This hexameric form can only be secreted if the NS1 protein is N-glycosylated. The NS1 protein has $2 \mathrm{~N}$-glycosylation sites. Introducing mutations at both sites in an infectious clone could thus impair NS1 protein hexamer secretion and its interaction with the complement $[100,101]$. Impairing NS1 protein interaction with the complement by mutating its $\mathrm{N}$-glycosylation sites may, in turn, lead to viral attenuation.

NS1 protein is not only detected as a hexamer, but as a dimer [102]. Impairing NS1 dimerization by mutating residue 250 in the Kunjin virus, WNV and MVEV impairs the replication of the resulting mutant virus. When injected into mice, the mutant virus NS1-P250L virulence is reduced [103-105]. DNA vaccine against WNV, based on this mutation, NS1-P250L, has also been tested in mice. Injection of a DNA plasmid, coding for the infectious full-length Kunjin virus genome and containing the mutation NS1-P250L, leads to the production of a mutant virus in the injected mice and induces protective immunity against Kunjin virus and WNV [106].

\section{Large-Scale Reencoding to Produce Attenuated Viruses}

New methods of viral attenuation exploiting the host cell machinery and its limits rather than host-virus interactions have been developed. For example, large-scale reencoding is a new method of attenuation that consists in mutating the viral genome without modifying the protein sequence by introducing silent mutations. Differences in codon usage may impair viral RNA translation and lead to viral attenuation [107].

This method was used randomly in a $1.4-\mathrm{kb}$ region of the TBEV NS5 protein coding sequence. Following reencoding, the virus obtained was attenuated in a mouse model of viral pathogenicity, without affecting viral fitness in vitro. It seems that reencoded viral RNA translation and replication were impaired. However, the reencoded virus is still potentially neuroinvasive as it could still be detected in the infected mouse brain. Thus, it is probably more prudent to use a less neurovirulent strain genome to develop a safe live attenuated reencoded vaccine [108].

The reencoding method can also be performed in order to optimize the codon usage. In a given host, synonymous codons are not used at the same frequency [109].

Intervirology 2017;60:8-18 
Table 1. Molecular tools and examples of attenuating mutations for designing new live attenuated vaccines

\begin{tabular}{|c|c|c|}
\hline Molecular tools & Examples of attenuating mutations & References \\
\hline $\begin{array}{l}\text { Chimerization in an attenuated } \\
\text { Flavivirus genome backbone }\end{array}$ & & $42-47,49-51,117$ \\
\hline $\begin{array}{l}\text { Chimerization in a measles virus } \\
\text { genome backbone }\end{array}$ & & $56,57,59,60$ \\
\hline $\begin{array}{l}\text { Chimerization in a nonintegrative } \\
\text { lentiviral vector }\end{array}$ & & 62,63 \\
\hline $\begin{array}{l}\text { Site-directed mutagenesis introducing } \\
\text { mutations identified in a live attenuated } \\
\text { Flavivirus vaccine }\end{array}$ & $\begin{array}{l}\text { WNV: E-L107F, E-E138K, E-Y172V, } \\
\text { E-T173A, E-A316V, E-K440R, } \\
\text { deletion of NS1' protein secretion } \\
\text { JEV: E-Q264H, M-I36F }\end{array}$ & $\begin{array}{l}30,43,68 \\
54,65\end{array}$ \\
\hline $\begin{array}{l}\text { Site-directed mutagenesis introducing } \\
\text { mutations impairing virus interactions with } \\
\text { the host cell }\end{array}$ & $\begin{array}{l}\text { JEV: NS5-E218A } \\
\text { WNV: NS2A-A30P, NS5-E218A } \\
\text { DV: NS5-E218A } \\
\text { TBEV: E-T310K } \\
\text { MVEV: E-D390N, E-D390E, E-390Y }\end{array}$ & $\begin{array}{l}75 \\
76,79 \\
76 \\
82 \\
83\end{array}$ \\
\hline $\begin{array}{l}\text { Passages of virus with an excess of cellular } \\
\text { receptors in order to impair E protein } \\
\text { interaction with its cellular receptor }\end{array}$ & JEV: E-G306E & 80 \\
\hline $\begin{array}{l}\text { Deleting prM, E, and NS1 protein } \\
\text { N-glycosylation sites }\end{array}$ & & $96-98$ \\
\hline Impair NS1 protein dimerization & $\begin{array}{l}\text { Kunjin virus: NS1-P250L } \\
\text { WNV: NS1-P250L } \\
\text { MVEV: NS1-P250L }\end{array}$ & $\begin{array}{l}103 \\
104 \\
105\end{array}$ \\
\hline Large-scale reencoding & & 108,110 \\
\hline Introduce miRNA target sequences & & 112 \\
\hline
\end{tabular}

The codon usage also differs, depending on the host. Thus, using codons that are less frequent in humans, but are still used in mosquitoes, can impair viral genome translation in human cells, but not in mosquito cells. This method was used on DV2 E and NS5 proteins. The resulting mutant viruses could replicate at the same level as the WT virus in mosquito cells, but their replication was impaired in mammalian cells. They were also attenuated in a mouse model of virus pathogenicity [110].

\section{Introducing MicroRNA Target Sequences into the Viral Genome}

A new method consisting of exploiting the antiviral activity of cellular microRNAs (miRNAs) is being explored. miRNAs are small RNAs that regulate many pathways by targeting mRNAs for cleavage or translational repression [111]. Introducing target sequences of miRNAs in the $3^{\prime}$ UTR region of a viral genome thus renders this genome sensitive to miRNA hybridization, leading to its cleavage or repression of its translation. For example, a highly virulent TBEV/DENV4 chimera was completely attenuated by introducing let-7c or miR124a miRNA target sequences. When miR-9 and miR128a miRNA target sequences were introduced, the resulting mutants were less attenuated than the other 2 mutants. However, it does not seem that this was due to a less efficient antiviral response of miR-9 and miR$128 \mathrm{a}$, but rather that the miRNA target sequence was mutated during viral replication [112]. Due to the lack of stability of an miRNA target sequence, this method should be combined with other methods of viral attenuation. 


\section{Lack of Vector Competence: Another Aspect to the Development of Live Attenuated Vaccines}

Flaviviruses are arboviruses maintained in a transmission cycle between mammalian hosts and arthropods. Due to the high rate of genomic mutations, vaccine candidates should not be able to be maintained in a transmission cycle, in order to limit the risks of reversion to virulent strains and thereby introducing and spreading pathogenic viruses in new geographical areas [113]. In this regard, the ability of some naturally occurring live attenuated strains of Flaviviruses (e.g., some strains of Kunjin virus) [114] to replicate in mosquitoes precludes their use as vaccine candidates.

The 2 live attenuated Flaviviruses used as vaccines, YFV-17D and JEV SA14-14-2, are not transmissible through the bite of mosquitoes [24, 115]. Thus, as for the mammalian attenuating mutations, identifying the mutations responsible for the inhibition of mosquito peripheral dissemination of the 17D and SA14-14-2 vaccine strains is important for the design of new molecularly cloned vaccines. So far, mutations in the M, E, NS2A, and NS4B proteins of the YFV 17D vaccine have been shown to be responsible for the inhibition of viral dissemination in mosquito organs $[67,116]$. When a chimeric Asibi/17D $\mathrm{M}-\mathrm{E}$ virus (encoding the YFV 17D M and E proteins in a YFV Asibi backbone) or the YFV 17D strain was given orally, less mosquitoes became infected and less virus was shed in their salivary glands than mosquitoes orally infected with YFV Asibi strain [67]. However, when YFV 17D or Asibi/17D M-E chimeric viruses were directly injected intrathoracically, mosquitoes shed the chimeric virus in their salivary glands at similar levels to YFV Asibi, showing that when the mosquito midgut epithelial barrier is bypassed, the YFV 17D strain and Asibi/17D M-E chimeric virus could infect the mosquito midgut cells, replicate in these cells, and disseminate to peripheral or- gans just like the YFV Asibi strain. Thus, it seems that mutations in the M and E proteins in YFV 17D impair viral replication in the mosquito midgut, reducing subsequent dissemination to the salivary glands [67].

\section{Conclusion}

Even though the fundamental bases for Flavivirus attenuation have still to be fully understood, it is now possible to design new generations of Flavivirus vaccine candidates using molecular biology technologies (Table 1). Via the construction of chimeric viruses, inspired by "old" empirically obtained, live attenuated viral strains, targeting the viral life cycle and its interactions with its host, introducing foreign cellular sequences, or even reencoding the viral genomes, or a combination of $\geq 2$ of these methods, we have now a wealth of possibilities to design attenuated Flaviviruses using infectious clones. However, not only viral attenuation and the induction of a protective immune response in a mammalian model of viral pathogenesis should be assessed, but also vector competence. Introducing mutations limiting vector infection or viral dissemination to the vector salivary glands may be compulsory in order to obtain a fully acceptable vaccine. As regulations regarding animal experimentation become more restrictive, identifying the key mutations required for attenuation as well as key features of genetic diversity prior to obtaining vaccine candidates will become vital in order to predict the potential attenuation of different, new-generation flaviviral vaccines without extensive animal experimentation.

\section{Disclosure Statement}

The authors have no conflict of interest to declare.

\section{References}

1 Weaver SC, Barrett ADT: Transmission cycles, host range, evolution and emergence of arboviral disease. Nat Rev Microbiol 2004;2: 789-801.

2 Solomon T, Dung NM, Kneen R, Gainsborough M, Vaughn DW, Khanh VT: Japanese encephalitis. J Neurol Neurosurg Psychiatry 2000;68:405-415.

3 Burki T: Yellow fever in Africa: a disaster waiting to happen. Lancet Infect Dis 2016;16: 896-897.
4 Rossi SL, Ross TM, Evans JD: West Nile Virus. Clin Lab Med 2010;30:47-65.

5 Wilder-Smith A, Gubler DJ, Weaver SC, Monath TP, Heymann DL, Scott TW: Epidemic arboviral diseases: priorities for research and public health. Lancet Infect Dis 2017;17:e101-e106.

6 Monath TP: Yellow fever vaccine. Expert Rev Vaccines 2005;4:553-574.
7 Yu Y: Phenotypic and genotypic characteristics of Japanese encephalitis attenuated live vaccine virus SA14-14-2 and their stabilities. Vaccine 2010;28:3635-3641.

8 Vaccines against tick-borne encephalitis: WHO position paper. Relev Epidemiol Hebd 2011;86:241-256.

9 Guy B, Guirakhoo F, Barban V, Higgs S, Monath TP, Lang J: Preclinical and clinical development of YFV 17D-based chimeric vaccines against dengue, West Nile and Japanese encephalitis viruses. Vaccine 2010;28:632-649.

Tools for Flavivirus Attenuation 
10 Guy B, Briand O, Lang J, Saville M, Jackson N: Development of the Sanofi Pasteur tetravalent dengue vaccine: one more step forward. Vaccine 2015;33:7100-7111.

11 Cantile C, Di Guardo G, Eleni C, Arispici M: Clinical and neuropathological features of West Nile virus equine encephalomyelitis in Italy. Equine Vet J 2000;32:31-35.

12 Steele KE, Linn MJ, Schoepp RJ, Komar N, Geisbert TW, Manduca RM, et al: Pathology of fatal West Nile virus infections in native and exotic birds during the 1999 outbreak in New York City, New York. Vet Pathol 2000; 37:208-224.

13 Sampson BA, Ambrosi C, Charlot A, Reiber K, Veress JF, Armbrustmacher V: The pathology of human West Nile Virus infection. Hum Pathol 2000;31:527-531.

14 Minke JM, Siger L, Karaca K, Austgen L, Gordy P, Bowen R, et al: Recombinant canarypoxvirus vaccine carrying the $\mathrm{prM} / \mathrm{E}$ genes of West Nile virus protects horses against a West Nile virus-mosquito challenge. Arch Virol Suppl 2004;18:221-230.

15 Centers for Disease Control and Prevention: West Nile virus disease cases and deaths reported to CDC by year and clinical presentation, 1999-2015. https://www.cdc.gov/westnile/resources/pdfs/data/1-WNV-DiseaseCases-by-Year_1999-2015_07072016.pdf (accessed 2016).

16 Chancey C, Grinev A, Volkova E, Rios M: The global ecology and epidemiology of West Nile virus. Biomed Res Int 2015;2015:376230.

17 Wikan N, Smith DR: Zika virus: history of a newly emerging arbovirus. Lancet Infect Dis 2016;16:e119-e126.

18 Zika - Epidemiological Update. Pan American Health Organization, 10 March 2017.

19 Gold CA, Josephson SA: Anticipating the challenges of Zika virus and the incidence of Guillain-Barré syndrome. JAMA Neurol 2016;73:905-906.

20 Lindenbach BD, Thiel HJ, Rice CM: Flaviviridae: the viruses and their replication; in Fields Virology, ed 5. Philadelphia, Lippincott-Raven, 2007, pp 1101-1152.

21 Eigen M: Viral quasispecies. Sci Am 1993;269: 42-49.

22 Domingo E: Quasispecies theory in virology. J Virol 2002;76:463-465.

23 Mateu MG, Martínez MA, Rocha E, Andreu D, Parejo J, Giralt E, et al: Implications of a quasispecies genome structure: effect of frequent, naturally occurring amino acid substitutions on the antigenicity of foot-and-mouth disease virus. Proc Natl Acad Sci USA 1989; 86:5883-5887.

$24 \mathrm{Yu}$ Y: Development of Japanese encephalitis attenuated live vaccine virus SA14-14-2 and its characteristics. InTech Open Science 2013 (chapt 11), DOI: 10.5772/52980.
25 Wang E, Ryman KD, Jennings AD, Wood DJ, Taffs F, Minor PD, et al: Comparison of the genomes of the wild-type French viscerotropic strain of yellow fever virus with its vaccine derivative French neurotropic vaccine. J Gen Virol 1995;76:2749-2755.

26 Beck A, Tesh RB, Wood TG, Widen SG, Ryman KD, Barrett ADT: Comparison of the live attenuated yellow fever vaccine 17D-204 strain to its virulent parental strain Asibi by deep sequencing. J Infect Dis 2014;209:334344.

27 Yun S-I, Song B-H, Polejaeva IA, Davies CJ, White KL, Lee Y-M: Comparison of the liveattenuated Japanese encephalitis vaccine SA14-14-2 strain with its pre-attenuated virulent parent SA14 strain: similarities and differences in vitro and in vivo. J Gen Virol 2016; 97:2575-2591.

28 Gromowski GD, Firestone C-Y, Whitehead SS: Genetic determinants of Japanese encephalitis virus vaccine strain SA14-14-2 that govern attenuation of virulence in mice. J Virol 2015;89:6328-6337.

29 Yun S-I, Song B-H, Kim J-K, Yun G-N, Lee E-Y, Li L, et al: A molecularly cloned, live-attenuated Japanese encephalitis vaccine SA1414-2 virus: a conserved single amino acid in the $i j$ Hairpin of the viral E glycoprotein determines neurovirulence in mice. PLoS Pathog 2014;10:e1004290.

30 Melian EB, Hinzman E, Nagasaki T, Firth AE, Wills NM, Nouwens AS, et al: NS1' of flaviviruses in the Japanese encephalitis virus serogroup is a product of ribosomal frameshifting and plays a role in viral neuroinvasiveness. J Virol 2010;84:1641-1647.

31 Ye Q, Li X-F, Zhao H, Li S-H, Deng Y-Q, Cao $\mathrm{R}-\mathrm{Y}$, et al: A single nucleotide mutation in NS2A of Japanese encephalitis-live vaccine virus (SA14-14-2) ablates NS1' formation and contributes to attenuation. J Gen Virol 2012; 93:1959-1964.

32 Wisseman CL, Sweet BH, Rosenzweig EC, Eylar OR: Attenuated living type 1 dengue vaccines. Am J Trop Med Hyg 1963;12:620-623.

33 Eckels $\mathrm{KH}$, Brandt WE, Harrison VR, McCown JM, Russell PK: Isolation of a temperature-sensitive dengue-2 virus under conditions suitable for vaccine development. Infect Immun 1976;14:1221-1227.

34 Bhamarapravati N, Yoksan S, Chayaniyayothin T, Angsubphakorn S, Bunyaratvej A: Immunization with a live attenuated dengue2 -virus candidate vaccine (16681-PDK 53): clinical, immunological and biological responses in adult volunteers. Bull World Health Organ 1987;65:189-195.

35 Angsubhakorn S, Yoksan S, Pradermwong A, Nitatpattana N, Sahaphong S, Bhamarapravatjz N: Dengue-3 (16562) PGMK 33 vaccine: neurovirulence, viremia and immune responses in Macaca fascicularis. Southeast Asian J Trop Med Public Health 1994;25:554559.
36 Halstead SB, Eckels KH, Putvatana R, Larsen LK, Marchette NJ: Selection of attenuated dengue 4 viruses by serial passage in primary kidney cells. IV. Characterization of a vaccine candidate in fetal rhesus lung cells. Am J Trop Med Hyg 1984;33:679-683.

37 Sangkawibha N, Rojanasuphot S, Ahandrik S, Viriyapongse S, Jatanasen S, Salitul V, et al: Risk factors in dengue shock syndrome: a prospective epidemiologic study in Rayong, Thailand. I. The 1980 outbreak. Am J Epidemiol 1984;120:653-669.

38 Morens DM, Venkateshan CN, Halstead SB: Dengue 4 virus monoclonal antibodies identify epitopes that mediate immune infection enhancement of dengue 2 viruses. J Gen Virol 1987;68:91-98.

39 Kanesa-thasan N, Sun W, Kim-Ahn G, Van Albert S, Putnak JR, King A, et al: Safety and immunogenicity of attenuated dengue virus vaccines (Aventis Pasteur) in human volunteers. Vaccine 2001;19:3179-3188.

40 Gould EA, Buckley A, Barrett ADT, Cammack N: Neutralizing (54K) and non-neutralizing (54K and $48 \mathrm{~K}$ ) monoclonal antibodies against structural and non-structural yellow fever virus proteins confer immunity in mice. J Gen Virol 1986;67:591-595.

41 Kaufman BM, Summers PL, Dubois DR, Houston Cohen W, Gentry MK, Timchak RL, et al: Monoclonal antibodies for dengue virus prM glycoprotein protect mice against lethal dengue infection. Am J Trop Med Hyg 1987; 41:576-580.

42 Chambers TJ, Nestorowicz ANN, Mason PW, Rice CM: Yellow fever/Japanese encephalitis chimeric viruses: construction and biological properties. J Virol 1999;73:3095-3101.

43 Arroyo J, Miller C, Catalan J, Myers GA, Ratterree MS, Trent DW, et al: ChimeriVax-West Nile virus live-attenuated vaccine: preclinical evaluation of safety, immunogenicity, and efficacy. J Virol 2004;78:12497-12507.

44 Li X-F, Deng Y-Q, Yang H-Q, Zhao H, Jiang $\mathrm{T}$, Yu X-D, et al: A chimeric dengue virus vaccine using Japanese encephalitis virus vaccine strain SA14-14-2 as backbone is immunogenic and protective against either parental virus in mice and nonhuman primates. J Virol 2013;87:13694-13705.

45 Li Z, Yang H, Yang J, Lin H, Wang W, Liu L, et al: Construction and preliminary investigation of a novel dengue serotype 4 chimeric virus using Japanese encephalitis vaccine strain SA14-14-2 as the backbone. Virus Res 2014; 191:10-20.

46 Yang H, Li Z, Lin H, Wang W, Yang J, Liu L, et al: A novel dengue virus serotype 1 vaccine candidate based on Japanese encephalitis virus vaccine strain SA14-14-2 as the backbone. Arch Virol 2016;161:1517-1526. 
47 Wang H-J, Li X-F, Ye Q, Li S-H, Deng Y-Q, Zhao $\mathrm{H}$, et al: Recombinant chimeric Japanese encephalitis virus/tick-borne encephalitis virus is attenuated and protective in mice. Vaccine 2014;32:949-956.

48 Men R, Bray M, Clark D, Chanock RM, Lai C-J: Dengue Type 4 virus mutants containing deletions in the 3J noncoding region of the RNA genome: analysis of growth restriction in cell culture and altered viremia pattern and immunogenicity in rhesus monkeys. J Virol 1996;70:3930-3937.

49 Huang CY, Butrapet S, Pierro DJ, Chang GJ, Hunt AR, Bhamarapravati N, et al: Chimeric dengue type 2 (vaccine strain PDK-53)/ dengue type 1 virus as a potential candidate dengue type 1 virus vaccine. J Virol 2000;74: 3020-3028.

50 Huang CY-H, Butrapet S, Tsuchiya KR, Bhamarapravati N, Gubler DJ, Kinney RM: Dengue 2 PDK-53 virus as a chimeric carrier for tetravalent dengue vaccine development. J Virol 2003;77:11436-11447.

51 Huang CY-H, Silengo SJ, Whiteman MC, Kinney RM: Chimeric dengue 2 PDK-53/ West Nile NY99 viruses retain the phenotypic attenuation markers of the candidate PDK53 vaccine virus and protect mice against lethal challenge with West Nile virus. J Virol 2005;79:7300-7310.

52 Pletnev AG, Bray M, Huggins J, Lai CJ: Construction and characterization of chimeric tickborne encephalitis/dengue type 4 viruses. Proc Natl Acad Sci USA 1992;89:10532-10536.

53 Pletnev AG, Putnak R, Speicher J, Wagar EJ, Vaughn DW: West Nile virus/dengue type 4 virus chimeras that are reduced in neurovirulence and peripheral virulence without loss of immunogenicity or protective efficacy. Proc Natl Acad Sci USA 2002;99:3036-3041.

54 Gromowski GD, Firestone C-Y, Hanson CT, Whitehead SS: Japanese encephalitis virus vaccine candidates generated by chimerization with dengue virus type 4 . Vaccine 2014; 32:3010-3018.

55 Lorin C, Combredet C, Labrousse V, Mollet $\mathrm{L}$, Desprès $\mathrm{P}$, Tangy F: A paediatric vaccination vector based on live attenuated measles vaccine. Therapie 2005;60:227-233.

56 Despres P, Combredet C, Frenkiel MP, Lorin C, Brahic M, Tangy F: Live measles vaccine expressing the secreted form of the West Nile virus envelope glycoprotein protects against West Nile virus encephalitis. J Infect Dis 2005; 191:207-214

57 Brandler S, Marianneau P, Loth P, Lacôte S, Combredet C, Frenkiel M-P, et al: Measles vaccine expressing the secreted form of West Nile virus envelope glycoprotein induces protective immunity in squirrel monkeys, a new model of West Nile virus infection. J Infect Dis 2012;206:212-219.

58 Crill WD, Roehrig JT: Monoclonal antibodies that bind to domain III of dengue virus $\mathrm{E}$ glycoprotein are the most efficient blockers of virus adsorption to Vero cells. J Virol 2001;75: 7769-7773.
59 Brandler S, Lucas-Hourani M, Moris A, Frenkiel M-P, Combredet C, Février M, et al: Pediatric measles vaccine expressing a dengue antigen induces durable serotype-specific neutralizing antibodies to dengue virus. PLoS Negl Trop Dis 2007;1:e96.

60 Brandler S, Ruffie C, Najburg V, Frenkiel $\mathrm{M}-\mathrm{P}$, Bedouelle $\mathrm{H}$, Desprès $\mathrm{P}$, et al: Pediatric measles vaccine expressing a dengue tetravalent antigen elicits neutralizing antibodies against all four dengue viruses. Vaccine 2010; 28:6730-6739.

61 Zennou V, Petit C, Guetard D, Nerhbass U, Montagnier L, Charneau P: HIV-1 Genome nuclear import is mediated by a central DNA flap. Cell 2000;101:173-185.

62 Iglesias MC, Frenkiel M-P, Mollier K, Souque $\mathrm{P}$, Desprès $\mathrm{P}$, Charneau $\mathrm{P}$ : A single immunization with a minute dose of a lentiviral vector-based vaccine is highly effective at eliciting protective humoral immunity against West Nile virus. J Gene Med 2006;8:265-274.

63 de Wispelaere M, Ricklin M, Souque P, Frenkiel M-P, Paulous S, Garcìa-Nicolàs $\mathrm{O}$, et al: A lentiviral vector expressing Japanese encephalitis virus-like particles elicits broad neutralizing antibody response in pigs. PLoS Negl Trop Dis 2015;9:e0004081.

64 Liang JJ, Liao CL, Liao JT, Lee YL, Lin YL: A Japanese encephalitis virus vaccine candidate strain is attenuated by decreasing its interferon antagonistic ability. Vaccine 2009;27: 2746-2754.

65 de Wispelaere M, Khou C, Frenkiel M, Desprès $\mathrm{P}$, Pardigon N: A single amino acid substitution in the $\mathrm{M}$ protein attenuates Japanese encephalitis virus in mammalian hosts. J Virol 2016;90:2676-2689.

66 Catteau A, Kalinina O, Wagner MC, Deubel V, Courageo MP, Desprès P: Dengue virus M protein contains a proapoptotic sequence referred to as ApoptoM. J Gen Virol 2003;84: 2781-2793.

67 McElroy KL, Tsetsarkin KA, Vanlandingham DL, Higgs S: Role of the yellow fever virus structural protein genes in viral dissemination from the Aedes aegypti mosquito midgut. J Gen Virol 2006;87:2993-3001.

68 Yu L, Robert Putnak J, Pletnev AG, Markoff L: Attenuated West Nile viruses bearing $3^{\prime} \mathrm{SL}$ and envelope gene substitution mutations. Vaccine 2008;26:5981-5988.

69 Kurane I, Meager A, Ennis FA: Induction of interferon alpha and gamma from human lymphocytes by dengue virus-infected cells. J Gen Virol 1986;67:1653-1661.

70 Diamond MS, Roberts TG, Edgil D, Lu B, Ernst J, Harris E: Modulation of dengue virus infection in human cells by alpha, beta, and gamma interferons. J Virol 2000;74:49574966.

71 Chang T, Liao C, Lin Y: Flavivirus induces interferon-beta gene expression through a pathway involving RIG-I-dependent IRF-3 and PI3K-dependent NF- $\mathrm{kB}$ activation 2006; $8: 157-171$.
72 Züst R, Cervantes-Barragan L, Habjan M, Maier R, Neuman BW, Ziebuhr J, et al: Ribose $2^{\prime}$-O-methylation provides a molecular signature for the distinction of self and non-self mRNA dependent on the RNA sensor Mda5. Nat Immunol 2011;12:137-143.

73 Issur M, Geiss BJ, Bougie I, Picard-Jean F, Despins S, Mayette J, et al: The flavivirus NS5 protein is a true RNA guanylyltransferase that catalyzes a two-step reaction to form the RNA cap structure. RNA 2009;15:2340-2350.

74 Kimura T, Katoh $\mathrm{H}$, Kayama $\mathrm{H}$, Saiga $\mathrm{H}$, Okuyama M, Okamoto T, et al: Ifit1 inhibits Japanese encephalitis virus replication through binding to $5^{\prime}$ capped 2'-O unmethylated RNA. J Virol 2013;87:9997-10003.

75 Li S-H, Dong H, Li X-F, Xie X, Zhao H, Deng $\mathrm{Y}-\mathrm{Q}$, et al: Rational design of a flavivirus vaccine by abolishing viral RNA $2^{\prime}-O$ methylation. J Virol 2013;87:5812-5819.

76 Zhou Y, Ray D, Zhao Y, Dong H, Ren S, Li Z, et al: Structure and function of flavivirus NS5 methyltransferase. J Virol 2007;81:38913903.

77 Liu WJ, Chen HB, Wang XJ, Huang H, Khromykh AA: Analysis of adaptive mutations in Kunjin virus replicon RNA reveals a novel role for the flavivirus nonstructural protein NS2A in inhibition of beta-interferon promoter-driven transcription. J Virol 2004;78: 12225-12235

78 Muñoz-Jordan JL, Sánchez-Burgos GG, Laurent-Rolle M, García-Sastre A: Inhibition of interferon signaling by dengue virus. Proc Natl Acad Sci 2003;100:14333-14338.

79 Liu WJ, Wang XJ, Clark DC, Lobigs M, Hall RA, Khromykh AA: A single amino acid substitution in the West Nile virus nonstructural protein NS2A disables its ability to inhibit al$\mathrm{pha} /$ beta interferon induction and attenuates virus virulence in mice. J Virol 2006;80:23962404.

$80 \mathrm{Ni} \mathrm{H}$, Barrett ADT: Attenuation of Japanese encephalitis virus by selection of its mouse brain membrane receptor preparation escape variants. Virology 1998;241:30-36.

81 Rey FA, Heinz FX, Mandl C, Kunz C, Harrison SC: The envelope glycoprotein from tickborne encephalitis virus at $2 \mathrm{~A}$ resolution. Nature 1995;375:291-298.

82 Mandl CW, Allison SL, Holzmann H, Meixner T, Heinz FX: Attenuation of tickborne encephalitis virus by structure-based site-specific mutagenesis of a putative flavivirus receptor binding site. J Virol 2000;74: 9601-9609.

83 Hurrelbrink RJ, McMinn PC: Attenuation of Murray Valley encephalitis virus by site-directed mutagenesis of the hinge and putative receptor-binding regions of the envelope protein. J Virol 2001;75:7692-7702.

84 Lee E, Lobigs M: Substitutions at the putative receptor-binding site of an encephalitic flavivirus alter virulence and host cell tropism and reveal a role for glycosaminoglycans in entry. J Virol 2000;74:8867-8875 
85 Lee E, Lobigs M: Mechanism of virulence attenuation of glycosaminoglycan-binding variants of Japanese encephalitis virus and Murray Valley encephalitis virus. J Virol 2002;76:4901-4911.

86 Lee E, Wright PJ, Davidson A, Lobigs M: Virulence attenuation of dengue virus due to augmented glycosaminoglycan-binding affinity and restriction in extraneural dissemination. J Gen Virol 2006;87:2791-2801.

87 Lee E, Lobigs M: E protein domain III determinants of yellow fever virus $17 \mathrm{D}$ vaccine strain enhance binding to glycosaminoglycans, impede virus spread, and attenuate virulence. J Virol 2008;82:6024-6033.

88 Zhao BT, Prince G, Horswood R, Eckels K, Summers P, Chanock R, et al: Expression of dengue virus structural proteins and nonstructural protein NS1 by a recombinant vaccinia virus. J Virol 1987;61:4019-4022.

89 Hanna SL, Pierson TC, Sanchez MD, Ahmed AA, Murtadha MM, Doms RW: N-linked glycosylation of West Nile virus envelope proteins influences particle assembly and infectivity. J Virol 2005;79:13262-13274.

90 Yoshii K, Yanagihara N, Ishizuka M, Sakai M, Kariwa $\mathrm{H}$ : N-linked glycan in tick-borne encephalitis virus envelope protein affects viral secretion in mammalian cells, but not in tick cells. J Gen Virol 2013;94:2249-2258.

91 Kim J-M, Yun S-I, Song B-H, Hahn Y-S, Lee $\mathrm{C}-\mathrm{H}, \mathrm{Oh} \mathrm{H}-\mathrm{W}$, et al: A single N-linked glycosylation site in the Japanese encephalitis virus prM protein is critical for cell type-specific prM protein biogenesis, virus particle release, and pathogenicity in mice. J Virol 2008;82: 7846-7862.

92 Zai J, Mei L, Wang C, Cao S, Fu ZF, Chen H, et al: $\mathrm{N}$-glycosylation of the premembrane protein of Japanese encephalitis virus is critical for folding of the envelope protein and assembly of virus-like particles. Acta Virol 2013; 57:27-33.

93 Beasley DWC, Whiteman MC, Zhang S, Huang CY-H, Schneider BS, Smith DR, et al: Envelope protein glycosylation status influences mouse neuroinvasion phenotype of genetic lineage 1 West Nile virus strains. J Virol 2005;79:8339-8347.

94 Yasui K: Neuropathogenesis of Japanese encephalitis virus. J Neurovirol 2002;8(suppl 2): 112-114.

95 Gamino V, Höfle U: Pathology and tissue tropism of natural West Nile virus infection in birds: a review. Vet Res 2013;44:39.

96 Shirato K, Miyoshi H, Goto A, Ako Y, Ueki T, Kariwa H, et al: Viral envelope protein glycosylation is a molecular determinant of the neuroinvasiveness of the New York strain of West Nile virus. J Gen Virol 2004;85:36373645 .
97 Whiteman MC, Li L, Wicker JA, Kinney RM, Huang C, Beasley DWC, et al: Development and characterization of non-glycosylated $\mathrm{E}$ and NS1 mutant viruses as a potential candidate vaccine for West Nile virus. Vaccine 2010;28:1075-1083.

98 Alsaleh K, Khou C, Frenkiel M, Lecollinet S, Vàzquez A, Ramírez E, et al: The E glycoprotein plays an essential role in the high pathogenicity of European - Mediterranean IS98 strain of West Nile virus. Virology 2016;492: 53-65.

99 Avirutnan P, Punyadee N, Noisakran S, Komoltri C, Thiemmeca S, Auethavornanan K, et al: Vascular leakage in severe dengue virus infections: a potential role for the nonstructural viral protein NS1 and complement. J Infect Dis 2006;193:1078-1088.

100 Somnuke P, Hauhart RE, Atkinson JP, Diamond MS, Avirutnan P: N-linked glycosylation of dengue virus NS1 protein modulates secretion, cell-surface expression, hexamer stability, and interactions with human complement. Virology 2011;413:253-264.

101 Flamand M, Megret F, Mathieu M, Lepault J, Rey FA, Deubel V: Dengue virus type 1 nonstructural glycoprotein NS1 is secreted from mammalian cells as a soluble hexamer in a glycosylation-dependent fashion. J Virol 1999;73:6104-6110.

102 Winkler G, Randolph VB, Cleaves GR, Ryan TE, Stollar V: Evidence that the mature form of the flavivirus nonstructural protein NS1 is a dimer. Virology 1988;162:187-196.

103 Hall RA, Khromykh AA, Mackenzie JM, Scherret JH, Khromykh TI, Mackenzie JS: Loss of dimerisation of the nonstructural protein NS1 of Kunjin virus delays viral replication and reduces virulence in mice, but still allows secretion of NS1. Virology 1999; 264:66-75.

104 Szentpáli-Gavallér K, Lim SM, Dencső L, Bányai K, Koraka P, Osterhaus ADME, et al: In vitro and in vivo evaluation of mutations in the NS region of lineage 2 West Nile virus associated with neuroinvasiveness in a mammalian model. Viruses 2016;8:49.

105 Clark DC, Lobigs M, Lee E, Howard MJ, Clark K, Blitvich BJ, et al: In situ reactions of monoclonal antibodies with a viable mutant of Murray Valley encephalitis virus reveal an absence of dimeric NS1 protein. J Gen Virol 2007;88:1175-1183.
106 Hall RA, Nisbet DJ, Pham KB, Pyke AT, Smith GA, Khromykh AA: DNA vaccine coding for the full-length infectious Kunjin virus RNA protects mice against the New York strain of West Nile virus. Proc Natl Acad Sci USA 2003;100:10460-10464.

107 Le Nouën C, Brock LG, Luongo C, McCarty T, Yang L, Mehedi M, et al: Attenuation of human respiratory syncytial virus by genome-scale codon-pair deoptimization. Proc Natl Acad Sci USA 2014;111:1316913174.

108 de Fabritus L, Nougairède A, Aubry F, Gould EA, de Lamballerie X, Gould E, et al: Attenuation of Tick-borne encephalitis virus using large-scale random codon re-encoding. PLoS Pathog 2015;11:e1004738.

109 Gutman GA, Hatfield GW: Nonrandom utilization of codon pairs in Escherichia coli. Proc Natl Acad Sci USA 1989;86:3699-3703.

110 Shen SH, Stauft CB, Gorbatsevych O, Song Y, Ward CB, Yurovsky A, et al: Large-scale recoding of an arbovirus genome to rebalance its insect versus mammalian preference. Proc Natl Acad Sci USA 2015;112: 4749-4754.

111 Bartel DP: MicroRNAs: genomics, biogenesis, mechanism, and function. Cell 2004;116: 281-297.

112 Heiss BL, Maximova OA, Pletnev AG: Insertion of microRNA targets into the flavivirus genome alters its highly neurovirulent phenotype. J Virol 2011;85:1464-1472.

113 Monath TP, Seligman SJ, Robertson JS, Guy B, Hayes EB, Condit RC, et al: Live virus vaccines based on a yellow fever vaccine backbone: standardized template with key considerations for a risk/benefit assessment. Vaccine 2015;33:62-72.

114 Beasley DWC, Li L, Suderman MT, Barrett ADT: Mouse neuroinvasive phenotype of West Nile virus strains varies depending upon virus genotype. Virology 2002;296: $17-23$.

115 Whitman L: Failure of Aedes aegypti to transmit yellow fever cultured virus (17D). Am J Trop Med 1939;19:19-26.

116 McElroy KL, Tsetsarkin KA, Vanlandingham DL, Higgs S: Manipulation of the yellow fever virus non-structural genes $2 \mathrm{~A}$ and $4 \mathrm{~B}$ and the $3^{\prime}$ non-coding region to evaluate genetic determinants of viral dissemination from the Aedes aegypti midgut. Am J Trop Med Hyg 2006; 75:1158-1164.

117 Guy B, Guirakhoo F, Barban V, Higgs S, Monath TP, Lang J: Preclinical and clinical development of YFV 17D-based chimeric vaccines against dengue, West Nile and Japanese encephalitis viruses. Vaccine 2010;28: 632-649. 